\section{Antipsychotic medication and venous thrombosis}

\author{
RENÉ THOMASSEN, JAN P. VANDENBROUCKE and FRITS R. ROSENDAAL
}

\author{
Background In an autopsy series, 10 \\ out of 27 deaths in which 'idiopathic' \\ pulmonary emboli were discerned as the \\ sole cause of death had occurred in \\ psychiatric patients.
}

Aims To investigate whether antipsychotic medication is a risk factor for venous thrombosis.

\section{Method A description of the 10}

psychiatric patients was obtained from the pulmonary emboli autopsy reports. We carried out a brief historic overview of the literature.We re-analysed data from the LeidenThrombophilia Study (LETS), a case-control study on patients with venous thrombosis.

Results In the autopsy reports, five out of I0 psychiatric patients with fatal pulmonary embolism had confirmed use of antipsychotic drugs. After the application of chlorpromazine and its analogues a higher incidence of venous thrombosis in psychiatric patients was described in the German literature between 1953 and 1977. In the re-analysis of the LETS case-control study, four patients used antipsychotic drugs versus none in the control group. Recent epidemiological studies of good methodological quality have confirmed these findings.

\section{Conclusions Venous thrombosis} appears to be associated with the use of antipsychotic drugs in psychiatric patients.

Declaration of interest None.
In a study to investigate a genetic basis for fatal pulmonary embolism in autopsy records at the Leiden University Medical Center, 10 out of 27 deaths in which pulmonary emboli were discerned as the sole cause of death and in which no other major underlying risk factor was present had occurred in psychiatric or psychogeriatric patients (Vandenbroucke et al, 1998). Inspired by this unanticipated finding, we searched the literature for studies on thrombosis in psychiatric patients. A number of papers, mostly in German literature between 1966 and 1984, reported an association between psychiatric illness and thrombosis, in particular pulmonary embolism. In 1997 this association received renewed attention in a study about mortality in people taking clozapine (Walker et al, 1997). In 2000, new studies reported a significantly increased risk of venous thrombosis in patients exposed to antipsychotic drugs (Hägg et al, 2000; Thomassen et al, 2000; Zornberg \& Jick, 2000).

We present a comprehensive report of the 10 psychiatric patients from the Leiden autopsy reports, and give a brief historic overview of the literature dealing with venous thrombosis in psychiatry. We also present secondary analyses of a case-control study on risk factors for venous thrombosis, the Leiden Thrombophilia Study (LETS; Koster et al, 1993).

\section{METHODS}

\section{Autopsy records}

At the Leiden University Medical Center, 14000 autopsy records from 1970 until mid-1994 were reviewed to select those in which pulmonary embolism was the sole cause of death. Only 27 autopsies fulfilled this criterion. Ten of these autopsies had occurred in psychiatric patients (Vandenbroucke et al, 1998). In order to find a common characteristic other than the psychiatric illness, we reviewed the original autopsy reports of the 10 psychiatric patients in detail. The autopsy reports included additional information such as a brief history of the admission or a copy of the discharge letter. We looked at age, gender, psychiatric diagnosis, hospital admission time, medication use, and clues for immobilisation and somatic illness prior to the pulmonary embolism.

\section{Historic literature overview}

Based on our findings in the autopsy record study we searched Medline from 1960 to 1998 for material on the subject of psychiatry and venous thrombosis, specifically antipsychotic medication and pulmonary embolism.

\section{Case-control study}

Details about the methodology of the casecontrol study have been published earlier (Koster et al, 1993). In brief, from 1990 to 1993,474 incident cases with a first episode of deep-vein thrombosis, in whom objective tests had confirmed the diagnoses, were selected from three anticoagulation clinics. In the Netherlands, anticoagulation clinics monitor coumarin treatment for virtually all patients diagnosed with venous thrombosis in well-defined geographic areas. The study was limited to outpatients. Psychiatric in-patients or general hospital in-patients who were in the hospital at the time of enrolment were excluded. The patients were asked to find a control subject of the same gender, about the same age, who was not a biological relative, had no history of thrombosis or malignant disorders and did not use coumarins. This population-based case-control study was used to estimate the risk of a first episode of deep-vein thrombosis in relation to several clotting factor abnormalities (Koster et al, 1993).

Participants were asked to report any medication use. Comorbidity and medical treatments were assessed. Risk factors for thrombosis such as oral contraceptive use, surgery, immobilisation and family history were evaluated extensively. For patients with a first episode of deep-vein thrombosis, medical reports on the admission and treatment were retrieved. Patients older than 70 years of age and patients with malignancies were excluded. Fifty-one patients declined participation and 19 patients were excluded because of psychiatric morbidity which would interfere with participation in the study (Koster et al, 1993). 
In the current re-analysis we investigated the frequency of daily use from the available records. First, we investigated the patient and control groups for any daily use of antipsychotics, antidepressants and benzodiazepines (including the index year in which the first episode of deep-vein thrombosis occurred). Second, patients who report usage of these drugs in the same year as the index date of their first thrombosis were excluded to be certain that the medication was used prior to the first thrombosis. Third, we restricted the analyses to those patients with mono-therapeutic medication use, to rule out any interference with comedication and comorbid conditions. Whether patients with a first episode of deep-vein thrombosis used more medication in general, compared with the controls, was checked for other commonly used medications such as beta-blockers and antacids. Odds ratios and $95 \%$ confidence intervals were generated through Epi Info 6 (Centers for Disease Control and Prevention \& World Health Organization, 1997).

\section{RESULTS}

\section{Autopsy records}

Among the 10 psychiatric patients there was a considerable diversity in psychiatric diagnosis and periods of admission. None of the patients had an acute psychotic episode prior to the pulmonary embolism. Five patients had used antipsychotic drugs. From the information we could extract from the autopsy reports this was the only common characteristic. Table 1 shows gender, age (mean and range) and presence of risk

Table I Antipsychotic medication, gender, mean age and risk factors for thrombosis of psychiatric patients with pulmonary embolism as the sole cause of death $(n=10)$

\begin{tabular}{lcc}
\hline & \multicolumn{2}{c}{ Antipsychotic medication } \\
\cline { 2 - 3 } & \multicolumn{1}{c}{ Yes $(n=5)$} & No $(n=5)$ \\
\hline Gender & 2 & - \\
Male & 3 & 5 \\
Female & $5 \mathrm{I}$ & $6 \mathrm{I}$ \\
Age (years) & & \\
Mean & $4 \mathrm{I}-67$ & $4 \mathrm{I}-67$ \\
Range & & 2 \\
Risk factors & 5 & 3 \\
None & - & \\
Yes (immobilisation) & & \\
\hline
\end{tabular}

factors in comparison with the patients who did not use antipsychotic drugs $(n=2)$ or for whom medication use was not reported $(n=3)$. The five patients who had taken antipsychotic medication were younger than those who had not, and no risk factors for pulmonary embolism were present in any of them. Among the non-antipsychotic users, three patients had impaired mobility. We concluded that the younger age at first occurrence as well as the lack of immobilisation pointed to an association of antipsychotic drug use and venous thrombosis.

\section{Historical literature overview}

The discovery of the antipsychotic qualities of chlorpromazine in 1953 was soon followed by several case reports in the German literature of fatal pulmonary embolism related to its use (Brehmer \& Ruckdeschel, 1953; Labhardt, 1954). A first case series, for the period 1954-1957, reported on 11 $(3.3 \%)$ cases of venous thrombosis and pulmonary embolism in a group of 338 phenothiazine users, compared with one in a non-phenothiazine control group (Grahmann \& Suchenwirth, 1959). In 1963, Mahmodian (1963) observed a three-fold increased risk of thrombosis in the period 1958-1961 in psychiatric and neurological patients compared with the years $1915-1922$ (from $6 \%$ to $18 \%$ in males and $10 \%$ to $29 \%$ in females). In 1966, Lal et al (1966) reported on consecutive autopsies on 357 psychiatric patients diagnosed mainly with schizophrenia and a chronic brain syndrome over the period 1961-1964. The prevalence of pulmonary embolism found in those autopsies was $10 \%$, which was the same as in all autopsies in the general hospital population (Lal et al, 1966). However, the occurrence of pulmonary embolism in the general hospital population is known to be high; so, the comparability with a general hospital population in fact points to a high incidence. In a similar study of 343 autopsy reports of psychiatric patients, Kendel \& Fodor (1969) found $98(29 \%)$ cases of pulmonary embolism, which in $16 \%$ was associated with acute psychiatric symptoms. Scholz (1967) studied 37 psychiatric patients with a pulmonary embolism as the clinical cause of death. Twenty-two patients had used antipsychotic drugs. They were younger and in one-third of the cases there was no explanatory comorbidity. Ziegler (1977) studied 688 autopsy reports with pulmonary embolism as the sole cause of death. Twenty-seven $(4 \%)$ had an underlying psychiatric disorder. Twelve suffered from schizophrenia and 15 had a depressive illness.

In a large observational study, MeierEwert et al (1967) compared two groups between 1953 and 1963: 1092 patients with schizophrenia or depression taking chlorpromazine, amitriptyline or imipramine and a group of 1172 similar psychiatric patients who did not use antipsychotic or antidepressant medication. The frequency of thrombo-embolic complications in the medication group was $2.9 \%$ versus $0.59 \%$ in the control group. However, 22 of the 34 medication users with a thrombo-embolic complication showed medical comorbidity. An interesting observation was made in 1984: five women with schizophrenia, 33-58 years of age, suffered from deep-venous thrombosis after an acute psychotic phase. They all died from pulmonary embolism without any comorbidity (Hindersin et al, 1984). Apart from general papers dealing with mortality in psychiatric hospitals (Licht et al, 1993; Hewer et al, 1995), until recently this 1984 paper was the last published paper on the specific subject of venous thrombosis in psychiatric patients. However, the subject found new attention in a study of Walker et al (1997) and Hägg et al (2000).

\section{Case-control analysis}

Baseline characteristics of out-patients with a first episode of venous thrombosis $(n=474)$ who used antipsychotic drugs, antidepressants or benzodiazepines and a matched control group $(n=474)$ are reported in Table 2. A hip operation, heart valve disease, heart rhythm abnormalities and intravenous heroin use until 1 year prior to the first episode of deep-vein thrombosis was reported as comorbidity. Medication use concerning five different medication categories among patients with a first episode of deep-vein thrombosis $(n=474)$ and the control group $(n=474)$ is shown in Table 3. In this table the medication use is reported with increasing restrictiveness: first, all use; second, use restricted to the year preceding the venous thrombosis; third, use limited to monotherapy. Four patients used antipsychotic drugs compared with none in the control group. Their mean age was 42.8 years. One of these patients also used an antidepressant and a benzodiazepine. The antipsychotic drugs used were levomepromazine, fluphenazine (both phenothiazines) and haloperidol. Antidepressant use differed between subjects in the case and control 
Table 2 Baseline characteristics of out-patients using antipsychotic drugs, antidepressants or benzodiazepines in cases with a first episode of deep-vein thrombosis $(n=474)$ and a control group $(n=474)$ during a period including the index year

\begin{tabular}{|c|c|c|c|c|c|c|}
\hline & \multicolumn{3}{|c|}{ Patients with deep-vein thrombosis } & \multicolumn{3}{|c|}{ Control } \\
\hline & Antipsychotics & Antidepressants & Benzodiazepines & Antipsychotics & Antidepressants & Benzodiazepines \\
\hline \multicolumn{7}{|l|}{ Gender } \\
\hline Male & 2 & 5 & 7 & - & 0 & 1 \\
\hline Female & 2 & 4 & 21 & - & 4 & 6 \\
\hline Mean age (years) & 42.8 & 51.8 & 49.0 & - & 41.5 & 60.3 \\
\hline \multicolumn{7}{|l|}{ Risk factors } \\
\hline Smoking & I & 4 & 19 & - & 2 & 6 \\
\hline Taking oral contraceptives & I & 0 & 0 & - & 1 & 0 \\
\hline Comorbidity & 1 & 3 & 7 & - & 0 & 1 \\
\hline Family history & 0 & 2 & 7 & - & 1 & 1 \\
\hline Total & 2 & 8 & 23 & - & 3 & 6 \\
\hline
\end{tabular}

groups and yielded an odds ratio of $2.3(95 \%$ CI 0.6-10.6). Medication use in general, such as beta-blocking agents and antacids, did not differ between patients and controls.

\section{DISCUSSION}

\section{Autopsy series}

A review of the Leiden University Hospital autopsy records in which pulmonary embolism was the sole cause of death led to the unanticipated finding of a large number of psychiatric patients with antipsychotic drug use. The finding that about one in three patients with idiopathic fatal pulmonary embolism were referred from a psychiatric institute was surprising because on average over the past 10 years the referral rate of such patients in autopsies was only 5$10 \%$. However, it cannot be excluded that selective factors leading to autopsy might have led to the large number of psychiatric patients in our series, as we discussed in our original report (Vandenbroucke et al,
1998). Because of this possible selection bias the hypothesis of antipsychotic drugs as a possible risk factor for deep-vein thrombosis was 'data-driven'.

\section{Historical literature overview}

Predominantly German literature between 1953 and 1984 confirmed a high prevalence of thrombosis in psychiatric patients, also with an association with antipsychotic use. Unfortunately, specific information about the diagnoses, medication use and physical circumstances under which the thrombosis occurred were often missing.

\section{Case-control analysis}

Our findings in the re-analysis of the casecontrol study are compatible with the hypothesis of antipsychotic drugs as a possible risk factor for deep-vein thrombosis. Moreover, the original study excluded all in-patients, also psychiatric in-patients, so that our conclusions relate only to outpatient antipsychotic drug use. As the numbers are small, confidence intervals are wide. In consequence the results in themselves are not conclusive.

\section{Recent literature}

Our results are in line with a 1992 publication reporting an impressive 17 -fold increase in risk of myocardial infarction observed among young women using psychotropic drugs and also an almost three-fold in creased risk of venous thrombo-embolism (Thorogood et al, 1992). A similar recent observation came from a study on mortality in clozapine users. Among current users of clozapine, 19 cases in 85399 person-years of fatal pulmonary embolisms were found. This risk was increased 5.2-fold when compared with the non-use of clozapine (Walker et al, 1997). Most recently, Zornberg \& Jick (2000) studied a baseline population of 29952 recipients of antipsychotic drugs. They found an odds ratio of 3.3 for high-potency antipsychotic drugs such as haloperidol and an odds ratio of

Table 3 Out-patients with a first episode of deep-vein thrombosis $(n=474)$ and a control group $(n=474)$ using psychotropic drugs, beta-blockers and antacid monotherapeutics during a period including the index year, excluding the index year and of monotherapy medication use only

\begin{tabular}{|c|c|c|c|c|c|c|c|c|c|}
\hline \multirow[t]{2}{*}{ Medication } & \multicolumn{3}{|c|}{ Including index year } & \multicolumn{3}{|c|}{ Excluding index year } & \multicolumn{3}{|c|}{ Monotherapy use excluding index year } \\
\hline & Case & Control & Odds ratio $(95 \% \mathrm{Cl})$ & Case & Control & Odds ratio $(95 \% \mathrm{Cl})$ & Case & Control & Odds ratio $(95 \% \mathrm{Cl})$ \\
\hline \multicolumn{10}{|l|}{ Psychotropic drugs } \\
\hline Antipsychotics & 4 & 0 & $(0.7-\sim)$ & 3 & 0 & $\infty(0.4-\sim)$ & 3 & 0 & $\infty(0.4-\sim)$ \\
\hline Antidepressants & 9 & 4 & $2.3(0.6-10.2)$ & 2 & 0 & $\infty(0.2-\sim)$ & 2 & 0 & $\infty(0.2-\sim)$ \\
\hline Benzodiazepines & 28 & 7 & $4.2(1.8-11.5)$ & 10 & 4 & $2.5(0.7-11.1)$ & 9 & 4 & $2.3(0.6-10.6)$ \\
\hline Beta-blockers & 25 & 20 & $1.3(0.7-2.4)$ & 16 & 9 & $1.8(0.7-4.5)$ & 12 & 9 & $1.3(0.5-3.5)$ \\
\hline Antacids & 16 & 10 & $1.6(0.7-3.9)$ & 7 & 4 & I.8(0.4-8.3) & 5 & 3 & $1.7(0.3-10.8)$ \\
\hline
\end{tabular}

$\infty$ denotes result of division by zero; $\sim$, not determined. 
24.1 for low-potency antipsychotic drugs such as chlorpromazine.

\section{Biological mechanism}

The biological mechanism explaining the relation between antipsychotic drugs and venous thrombosis is unknown. Multiple hypotheses have been postulated. In the most recent publications of Zornberg \& Jick (2000) and Hägg et al (2000) three possible mechanisms are discussed: antipsychotic drugs enhance aggregation of platelets; anticardiolipin antibodies are associated with increased risk of thrombosis and their levels are raised in some patients using chlorpromazine; and venous stasis is exacerbated by sedation. An alternative explanation can be found in the article of Hindersin et al (1984): the acute psychotic phase is associated with an increase of adrenaline secretion, which enhances the coagulation mechanism.

Venous thrombosis appears to be associated with the use of antipsychotic drugs in psychiatric patients. Clinicians should be aware of this possible relation when patients using antipsychotic drugs have complaints such as chest pain or dyspnoea. However, it cannot be completely excluded that the findings on antipsychotic drugs are an expression of some other underlying risk factors of the disease itself. Also, the use of these drugs might predispose to venous thrombosis because of other medication effects such as sedation and immobilisation. Because of the clinical implications we conclude that the association between venous thrombosis and psychiatric illness is worthy of renewed investigation, with an emphasis on the mechanisms that might elucidate a direct effect of antipsychotic drugs.

\section{REFERENCES}

Brehmer, G. \& Ruckdeschel, K. T. (1953) Zur Technik der Winterschlafbehandlung. Deutsche medizinische Wochenschrift, 78, 1724-1725.

\section{Centers for Disease Control and Prevention \&} World Health Organization (1997) Epi Info 6. Version 6.04b. A Word Processing Database and Statistical Program for Public Health. Geneva: Centers for Disease Control and Prevention (CDC) USA \& World Health Organization

Grahmann, H. \& Suchenwirth, R. (1959) Thrombose hazard in chlorpromazine and reserpine therapy of endogenous psychosis. Nervenarzt, 30, 224-225.

Hägg, S., Spigset, O. \& Söderström, T. G. (2000) Association of venous thromboembolism and clozapine. Lancet, 355, II55-1156.

Hewer, W., Rossier, W., Falkenheuer, B., et al (1995) Mortality among patients in psychiatric hospitals in Germany. Acta Psychiatrica Scandinavica, 91, 174-179.

\section{CLINICAL IMPLICATIONS}

A higher incidence of venous thrombosis in psychiatric patients might be associated with the use of antipsychotic medication.

- When a patient uses antipsychotic drugs in the presence of risk factors for venous thrombo-embolism, the attending physician should be aware of the increased risk of venous thrombosis.

- The association between venous thrombosis and antipsychotic medication should be studied in investigations specifically designed to elucidate mechanisms by which the use of antipsychotics leads to venous thrombosis.

\section{LIMITATIONS}

- The associations between venous thrombosis and the use of antipsychotic drugs might be an expression of other underlying risk factors.

- The original research on which our hypothesis is based was not designed for its investigation.

- The biological mechanism to explain the possible association between antipsychotic drugs and venous thrombo-embolism is unknown.

RENÉ THOMASSEN, MD, Department of Psychiatry, JAN P.VANDENBROUCKE, PhD, Department of Clinical Epidemiology, FRITS R. ROSENDAAL, MD, Department of Clinical Epidemiology and Thrombosis and Haemostasis Center, Leiden University Medical Center, The Netherlands

Correspondence: Jan P. Vandenbroucke, Leiden University Medical Center, Department of Clinical Epidemiology, PO Box 9600, 2300 RC Leiden, The Netherlands. Tel.: +31 7I 5265230; Fax: +31 7 5248122; e-mail:vdbroucke@mail.medfac.leidenuniv.nl

(First received I3 July 2000, final revision 22 January 200I, accepted 23 January 200I)

Hindersin, P., Siegmund, R. \& Körting, H. J. (1984) Thrombophile diathesen als Hämostasestörungen bei akuten psychosen. Psychiatrie, Neurologie und medizinsche Psycholgie, 36, 702-709.

Kendel, K. \& Fodor, S. (1969) Pulmonary embolism and symptomatic psychosis. German Medical Monthly, I4, 184-187.

Koster, T., Rosendaal, F., Ronde de, H., et al (1993) Venous thrombosis due to poor anticoagulant response to activated protein C: Leiden thrombophilia study. Lancet, 342, I503-1506.

Labhardt, E. (1954) Technik, Nebenerscheinungen und Komplikationen der Largactil Therapie. Schweizer Archives Neurologie und Psychiatrie, 73, 338-344.

Lal, S., Bleiman, M., Brown, B. N., et al (1966)

Pulmonary embolism in psychiatric patients. Journal of the American Geriatrics Society, 14, ||38-||43.

Licht, R.W., Mortensen, P. B., Gouliaev, G., et al (1993) Mortality in Danish psychiatric long-stay patients, 1972-1982. Acta Psychiatrica Scandinavica, 87, 336-341.

Mahmodian, M. H. (1963) Ursachen der Lungenembolie bei psychisch und neurologisch Kranken. Deutsche medizinische Wochenschrift, 204, 229-244.

Meier-Ewert, K., Baumgart, H. H. \& Friedenberg, P. (1967) Thromboembolische Komplikationen bei neuro- und thymoleptischer Behandlung. Deutsche medizinische Wochenschrift, $92,2174-2178$.

Scholz, V. (1967) Über thromboembolische Komplicationen unter neuroleptischer Medikation. Nervenartz, 38, 174-177.

Thomassen, R., Vandenbroucke, J. P. \& Rosendaal, F. R. (2000) Antipsychotic drugs and venous thromboembolism. Lancet, 365, 252.

Thorogood, M., Cowen, P., Mann, J., et al (1992) Fata myocardial infarction and use of psychotropic drugs in young women. Lancet, 340, 1067-1068.

Vandenbroucke, J. P., Bertina, R. M., Holmes, Z. R., et al (1998) Factor $V$ Leiden and fatal pulmonary embolism. Thrombosis and Haemostasis, 79, 51।-516.

Walker, A., Lanza, L. \& Rothman, K. (1997) Mortality in current and former users of clozapine. Epidemiology, 8, 67I-677.

Ziegler, H. K. (1977) Lungenembolie aus der sicht des Pathologen. Medizinische Klinik, 72, 1063-1070.

Zornberg, G. L. \& Jick, H. (2000) Antipsychotic drug use and the risk of first-time idiopathic venous thromboembolism: a case-control study. Lancet, 356 , 1219-1223. 